\title{
Antibacterial activity of oxyresveratrol against methicillin-resistant Staphylococcus aureus and its mechanism
}

\author{
DAE-KI JOUNG ${ }^{1 *}$, SU-HYUN MUN ${ }^{1 *}$, SUNG-HOON CHOI $^{1}$, OK-HWA KANG ${ }^{1}$, SUNG-BAE KIM $^{1}$, \\ YOUNG-SEOB LEE ${ }^{2}$, TIAN ZHOU ${ }^{1}$, RYONG KONG ${ }^{1}$, JANG-GI CHOI ${ }^{1}$, DONG-WON SHIN ${ }^{3}$, \\ YOUN-CHUL KIM ${ }^{4}$, DONG-SUNG LEE ${ }^{4}$ and DONG-YEUL KWON ${ }^{1}$
}

\author{
${ }^{1}$ Department of Oriental Pharmacy, College of Pharmacy, Wonkwang-Oriental Medicines Research Institute, Institute of \\ Biotechnology, Wonkwang University, Iksan, Jeonbuk 570-749; ${ }^{2}$ Department of Herbal Crop Research, National Institute of \\ Horticultural and Herbal Science, Rural Development Administration, Eumsung, Chungbuk 369-873; \\ ${ }^{3}$ Department of Oriental Medicine Resources, Sunchon National University, Suncheon, Jeonnam 540-742; \\ ${ }^{4}$ Standardized Material Bank for New Botanical Drugs, College of Pharmacy, \\ Wonkwang University, Iksan, Jeonbuk 570-749, Republic of Korea
}

Received October 23, 2014; Accepted October 22, 2015

DOI: $10.3892 / \mathrm{etm} .2016 .3486$

\begin{abstract}
Oxyresveratrol (ORV) is a naturally occurring compound found in mulberries that exhibits a wide spectrum of biological activities. However, the underlying mechanism of the action of ORV against the methicillin-resistant S. aureus (MRSA) pathogen has not yet been reported. MRSA is multidrug-resistant, causing skin and other types of infections. The aim of the present study was to examine the antimicrobial activity of ORV and the underlying mechanism of its action on MRSA. The antibacterial activity of ORV was evaluated using a minimum inhibitory concentration (MIC) assay, and the mechanism of its antibacterial action on $S$. aureus was investigated using a combination of ORV with detergent, ATPase inhibitors and peptidoglycan (PGN). In addition, the survival characteristics and changes in MRSA morphology were monitored using transmission electron microscopy (TEM). The MIC value of ORV against all S. aureus strains was found to be $125 \mu \mathrm{g} / \mathrm{ml}$. The optical density at $600 \mathrm{~nm}$ of each suspension treated using a combination of ORV with Triton X-100, $N, N^{\prime}$-dicyclohexylcarbodiimide or sodium azide was reduced by $68.9-89.8 \%$ compared with the value upon treatment with ORV alone. In the ORV and PGN combination assay, direct binding of ORV with PGN from S. aureus was
\end{abstract}

Correspondence to: Professor Dong-Yeul Kwon, Department of Oriental Pharmacy, College of Pharmacy, Wonkwang-Oriental Medicines Research Institute, Institute of Biotechnology, Wonkwang University, 344-2 Sinyong Dong, Iksan, Jeonbuk 570-749, Republic of Korea

E-mail: sssimi@wku.ac.kr

${ }^{*}$ Contributed equally

Key words: oxyresveratrol, antibacterial mechanism, membrane permeability, methicillin-resistant Staphylococcus aureus, ATPase evident. Furthermore, TEM examination of MRSA treated with ORV showed alterations in septa formation. In conclusion, these results showed that ORV has a strong antibacterial effect against $S$. aureus, mainly by increasing membrane permeability and inhibiting ATPase when combined with other drugs.

\section{Introduction}

Methicillin-resistant Staphylococcus aureus (MRSA) is a prominent human pathogen that is known for causing skin infections, as well as hospital-acquired pneumonia, osteomyelitis and abscesses (1); MRSA may also result in considerable morbidity and mortality in orthopedic patients. The mortality rate of MRSA bacteremia is twice as high as that of patients with methicillin-sensitive Staphylococcus aureus (MSSA) (2). In addition, the complication rate and cost of periprosthetic joint infection due to MRSA is considerably higher compared with that in MSSA infection (3). Patients receiving orthopedic implants are highly vulnerable due to the possibility of biofilm formation and long-term morbidity $(4,5)$. Currently, the incidence of MRSA in orthopedic departments is increasing (6). MRSA strains are resistant not only to $\beta$-lactam antibiotics, but also to fluoroquinolones and other families of antibiotics (7).

The Morus genus is part of the Moraceae family, which includes 10-16 species of deciduous trees that are found worldwide (8). For >1,900 years, different parts of the Morus plants, including the branches, fruit, leaves, bark, shoot and root, have been used in China as food and herbal medicine (9). The compounds resveratrol and oxyresveratrol (ORV) are present in the Morus plants and have been revealed to possess antioxidant activity (10). A previous study reported that ORV inhibited the production of prostaglandin $\mathrm{E}_{2}$ and nitrogen oxide (NO), the expression of inducible NO synthase (iNOS) and the activation of nuclear factor- $\kappa \mathrm{B}$ in macrophages, while consistently reducing carrageenan-induced edema in a mouse model (11). 
ORV, a polyphenolic stilbene, is abundantly derived from the heartwood of the traditional Thai plant, Artocarpus lakoocha Roxburgh, which belongs to the Moraceae family $(12,13)$. This compound has been demonstrated to have an inhibitory effect on the growth of Herpes simplex virus (HSV)-1 and HSV-2 wild types, drug-resistant HSV-1 strains (14), clinical isolates of HSV-1 and HSV-2 (15), in addition to numerous varicella zoster virus (VZV) strains, including the wild type, thymidine kinase-deficient and DNA polymerase VZV mutants in vitro (16). Numerous biological activities of ORV have also been reported, including tyrosinase-inhibition (17) and antioxidant (18) and anthelmintic activities (19). Topical application of $30 \% w / w$ ORV in petroleum jelly suspension was reported to provide superior therapeutic efficacy compared with oral treatment with ORV for cutaneous HSV-1 infection in mice (14).

However, the antibacterial capacity and mechanism of ORV against Staphylococcus aureus (S. aureus) remain unknown. Therefore, the present study investigated the antibacterial activities of ORV alone or in combination with bacterial membrane-binding agents, including Triton X-100 (TX), sodium azide $\left(\mathrm{NaN}_{3}\right)$ and $N, N^{\prime}$-dicyclohexylcarbodiimide (DCCD). In addition, the effects of adding peptidoglycan (PGN; derived from S. aureus) into Müller-Hinton broth (MHB) containing ORV alone were also examined. The aim of the present study was to gain an insight into the antibacterial activity, survival characteristics and changes in the bacterial morphology and mechanism of ORV against MRSA.

\section{Materials and methods}

Isolation and purification of ORV. ORV (purity, >96.32\%) was provided by the Standardized Material Bank for New Botanical Drugs (no. NNMBP000018) at Wonkwang University (Iksan, Korea). Twigs from the Morus alba plant were supplied by the Herbal Medicine Co-operative Association of Jeonbuk Province (Iksan, Korea) in October 2010. EtOH (2 litres) was added to $2 \mathrm{~kg}$ of dried Morus alba twigs for 20 days at room temperature. The dried residue of the EtOH extract (101 g) was dissolved in $40 \%$ aqueous $\mathrm{MeOH}$ (1 1) and separated with $n$-hexane ( $800 \mathrm{ml}$, twice), $\mathrm{CH}_{2} \mathrm{Cl}_{2}$ (800 $\mathrm{ml}$, twice) and EtOAc $(800 \mathrm{ml}$, twice), successively. A column $(5 \times 16 \mathrm{~cm})$ filled with Sephadex LH-20 (GE Healthcare Bio-Sciences, Pittsburgh, PA, USA) was used to perform chromatography on the soluble fraction of $\mathrm{CH}_{2} \mathrm{Cl}_{2}(8.53 \mathrm{~g}) ; \mathrm{CH}_{2} \mathrm{Cl}_{2}-\mathrm{MeOH}$ (ratio, 4:1 to $1: 1$, for each volume of $300 \mathrm{ml}$ ) was used to obtain four fractions (denoted A-D). Next, the soluble fraction of EtOAc (4.83 g) was separated by chromatography on a silica gel $\left(250 \mathrm{~g}\right.$ ) column using $\mathrm{CH}_{2} \mathrm{Cl}_{2}-\mathrm{MeOH}$ (ratio, 8:1 to $4: 1$, for each volume of $600 \mathrm{ml}$ ) in order to obtain three additional fractions (denoted E-G). Subsequently, a silica gel (150 g) column (eluent, $n$-hexane-acetone, at a ratio of 1:1) was used to perform chromatography on fraction E (2.77 g), a fraction chosen due to its abundance of ORV. The sample was further purified by Sephadex LH-20 column chromatography (2.5x20 cm; eluent, $\mathrm{CH}_{2} \mathrm{Cl}_{2}-\mathrm{MeOH}$, at a ratio of 4:1) in order to obtain ORV (1.12 g, 0.056\% w/w). The structure of ORV was then identified by mass spectrometry [using a Q-TOF micro LC-MS/MS instrument (Waters Corporation, Manchester, UK) located at Korea University, Seoul, Korea] and nuclear
Table I. Determination of the mecA gene status of the S. aureus strains used.

\begin{tabular}{lcccc}
\hline S. aureus strain & Class & $\begin{array}{c}\text { mecA } \\
\text { gene }\end{array}$ & $\begin{array}{c}\beta \text {-lactamase } \\
\text { activity }\end{array}$ & $\begin{array}{c}\text { Antibiotic } \\
\text { resistance }\end{array}$ \\
\hline ATCC 33591 & MRSA & + & + & AM, OX \\
ATCC 25923 & MSSA & - & - & - \\
Clinical isolates & & & & \\
DPS-1 & MRSA & + & + & AM, OX \\
DPS-2 & MRSA & + & - & AM, OX \\
DPS-3 & MRSA & + & + & AM, OX \\
\hline
\end{tabular}

S. aureus, Staphylococcus aureus; ATCC, American Type Culture Collection; MRSA, methicillin-resistant S. aureus; AM, ampicillin; OX, oxacillin; MSSA, methicillin-sensitive S. aureus; DPS, strain from the Department of Plastic Surgery, Wonkwang University Hospital.

magnetic resonance analyses [recorded in $\mathrm{CDCl}_{3}$ or acetone- $\mathrm{d}_{6}$ using a JNM ECX-400 spectrometer (JEOL, Ltd., Tokyo, Japan) operating at $400 \mathrm{MHz}$ for ${ }^{1} \mathrm{H}$ and at $100 \mathrm{MHz}$ for $\left.{ }^{13} \mathrm{C}\right]$ in accordance with our previous study (20).

Reagents. Müeller-Hinton agar (MHA) and MHB, as nutrient media, were purchased from BD Biosciences (Franklin Lakes, NJ, USA). $\mathrm{NaN}_{3}$ and PGN were obtained from Sigma-Aldrich (Buchs, Switzerland) and TX, DCCD, purified lipopolysaccharide (LPS), ampicillin (AM), oxacillin (OX), gentamicin (GT), vancomycin (VC), norfloxacin (NR) and ciprofloxacin (CP) were obtained from Sigma-Aldrich (St. Louis, MO, USA).

Bacterial strains and growth conditions. Three clinical isolates of MRSA (DPS-1, -2 and -3) were obtained from three different patients of the Department of Plastic Surgery, Wonkwang University Hospital (Iksan, Korea), in accordance with the methods used in a previous study (1). Two additional strains were purchased from the American Type Culture Collection (ATCC; Manassas, VA, USA); these were the S. aureus strains ATCC 33591 (methicillin-resistant) and ATCC 25923 (methicillin-susceptible) (Table I). Prior to the experiments, all strains were stored in $30 \%$ glycerol and frozen at $-70^{\circ} \mathrm{C}$. The bacterial strains were inoculated onto MHA plates and incubated at $37^{\circ} \mathrm{C}$ for $24 \mathrm{~h}$.

Antimicrobial resistance testing. Testing for mecA gene activity was used to identify staphylococci resistant to $\beta$-lactam antibiotics (21). Detection of the mecA gene in MRSA strains (Table I) was performed by polymerase chain reaction amplification, as follows: Prior to DNA extraction, bacteria stock cultures were subcultured twice onto MHA plates. For rapid extraction, 1-5 bacterial colonies were suspended in $300 \mu \mathrm{l}$ of buffer from the Easy-RED BYF total RNA extraction kit (Intron Biotechnology, Inc., Seongnam, Korea) and heated at $100^{\circ} \mathrm{C}$ for $20 \mathrm{~min}$. After centrifugation at $10,000 \mathrm{x} \mathrm{g}$ for $10 \mathrm{~min}, 2 \mu \mathrm{l}$ of the supernatant was used for the DNA extraction. cDNA was synthesized from RNA at $42^{\circ} \mathrm{C}$ for 60 min using a Power cDNA synthesis kit (Intron Biotechnology, Inc.). PCR reactions were performed using $2 \mu \mathrm{l}$ 
Table II. S. aureus strains used in the experiments and MIC.

\begin{tabular}{|c|c|c|c|c|c|c|c|c|}
\hline \multirow[b]{2}{*}{ S. aureus strain } & \multirow[b]{2}{*}{ Class } & \multicolumn{7}{|c|}{$\operatorname{MIC}(\mu \mathrm{g} / \mathrm{ml})$} \\
\hline & & ORV & $\mathrm{AM}$ & OX & $\mathrm{VC}$ & GT & NR & $\mathrm{CP}$ \\
\hline ATCC 25923 & MSSA & 125 & 31.25 & 125 & 3.9 & 62.5 & 15.6 & 31.25 \\
\hline ATCC 33591 & MRSA & 125 & 1,000 & 500 & 1.95 & 31.25 & 250 & 500 \\
\hline DPS-1 & MRSA & 125 & 1,000 & 1,000 & 1.95 & 250 & 31.25 & 125 \\
\hline DPS-2 & MRSA & 125 & 1,000 & 1,000 & 3.9 & 125 & 31.25 & 125 \\
\hline DPS-3 & MRSA & 125 & 1,000 & 1,000 & 1.95 & 125 & 31.25 & 125 \\
\hline
\end{tabular}

S. aureus, Staphylococcus aureus; MIC, minimum inhibitory concentration; ORV, oxyresveratrol; AM, ampicillin; OX, oxacillin; VC, vancomycin; GT, gentamicin; NR, norfloxacin; CP, ciprofloxacin; ATCC, American Type Culture Collection; MSSA, methicillin-sensitive S. aureus; MRSA, methicillin-resistant S. aureus; DPS, strain from the Department of Plastic Surgery, Wonkwang University Hospital.

of cDNA per reaction and an MRSA Primer mix kit (Genotek Co., Daejeon, Korea) in a total reaction volume of $20 \mu 1$. The PCR amplification consisted of 30 cycles $\left(94^{\circ} \mathrm{C}, 60 \mathrm{sec} ; 55^{\circ} \mathrm{C}\right.$, $\left.60 \mathrm{sec} ; 72^{\circ} \mathrm{C}, 60 \mathrm{sec}\right)$. The final PCR products were separated on a $2 \%$ agarose gel. $\beta$-lactamase activity, indicating antibiotic resistance, was determined using a $\beta$-lactamase activity assay kit (Sigma-Aldrich), according to the manufacturer's protocol.

Antibacterial susceptibility experiments. The minimum inhibitory concentration (MIC) was determined using a broth micro-dilution method, in accordance with the Clinical and Laboratory Standards Institute guidelines (22). Briefly, an inoculum of the microorganisms from the MHA plates was prepared by growing the microorganism in a two-fold dilution of ORV in MHB for $24 \mathrm{~h}$. Next, the suspension was adjusted to a 0.5 McFarland standard turbidity $\left[\sim 1.5 \times 10^{8}\right.$ colony-forming units $(\mathrm{CFU}) / \mathrm{ml}$, with the final inoculums adjusted to $1.5 \times 10^{6} \mathrm{CFU} / \mathrm{ml}$. The MIC was defined as the lowest concentration of ORV and the various antibiotics that inhibited microorganism growth following incubation at $37^{\circ} \mathrm{C}$ for $24 \mathrm{~h}$ in well plates. Subsequent to the incubation period, the well plates were visually examined for turbidity. Cloudiness indicated that bacterial growth had not been inhibited by the concentration of antimicrobial agent contained in the medium. The antibiotics AM, OX, GT, VC, NR and CP were used in comparisons of MIC with ORV-only conditions (Table II) (21).

Antibacterial activity with detergent or ATPase inhibitors. The antibacterial activity of ORV in the presence of a detergent, TX, was analyzed to elucidate whether the antibacterial activity of ORV was associated with altered membrane permeability. The activity of ORV in the presence of ATPase-inhibiting agents, DCCD and $\mathrm{NaN}_{3}$, was also examined to determine whether it was associated with multidrug resistance. In order to determine the detergent-induced permeabilization, ORV was mixed with TX (23), since the non-ionic detergent TX is known to greatly increase antibiotic sensitivity (24). DCCD and $\mathrm{NaN}_{3}$, two metabolic inhibitors that decrease the ATP levels by disrupting electrochemical proton gradients in bacteria $(25,26)$, were used as ATPase inhibitors. The antibacterial activity of $62.5 \mu \mathrm{g} / \mathrm{ml}$ ORV was measured in the presence of $0.1 \% \mathrm{TX}, 0.001 \% \mathrm{NaN}_{3}$ and $6.25 \mu \mathrm{g} / \mathrm{ml}$ DCCD compared to that of ORV alone, determined by a reading of absorbance [or optical density measured at a wavelength of $\left.600 \mathrm{~nm}\left(\mathrm{OD}_{600}\right)\right]$ using an Epoch microplate spectrophotometer (Bio-Tek Instruments, Inc., Winooski, VT, USA). This measurement was indicative of cell proliferation.

Effect of exogenous PGN on ORV activity. ORV may bind to the cell wall and disrupt its integrity. To confirm the action of ORV upon addition of exogenous PGN, ORV + PGN combination assays were performed using the method described by Zhao et al (27). These assays were used to determine whether ORV directly binds to PGN or disrupts the integrity of the cell wall when the same concentrations of ORV and PGN were combined (0-62.5 $\mu \mathrm{g} / \mathrm{ml}$, i.e. up to $50 \%$ of the MIC of ORV). LPS was used as a control.

Transmission electron microscopy (TEM). Exponential-phase MRSA cultures were prepared using overnight cultures incubated in MHB at $37^{\circ} \mathrm{C}$ until they reached the mid-logarithmic phase of growth (21). Subsequently, the MHB-grown exponential-phase MRSA cultures were treated with ORV at 50\% of the MIC, and at the MIC dose for $30 \mathrm{~min}$. Subsequent to the treatment, $2 \mathrm{ml}$ of the culture was collected by centrifugation at $10,000 \mathrm{x}$ g for $10 \mathrm{~min}$. Following removal of the supernatant, cell pellets were fixed with modified Karnovsky's fixative (Electron Microscopy Sciences, Hatfield, PA, USA). Energy-filtering TEM (Libra 120; Carl Zeiss, Oberkochen, Germany) was then performed to examine the samples at an accelerating voltage of $120 \mathrm{kV}$. Transmitted electron signals were recorded using a $4 \mathrm{k} \mathrm{x} 4 \mathrm{k}$ pixel slow-scan charge-coupled device camera (Ultrascan 4000 SP; Gatan Inc., Pleasanton, CA, USA) attached to the electron microscope.

Statistical analysis. All experiments were performed three times. Data from the experiments are presented as the mean \pm SEM. Dunnett's t-test was used for multiple comparisons. $P$-values $<0.01$ were considered to represent a statistically significant difference.

\section{Results and Discussion}

Antibacterial agents inhibit bacterial growth through a variety of complex mechanisms, including the disruption of cell membranes, the inhibition of cell wall, nucleic acid and protein synthesis and the inhibition of nucleic acid metabolism (28). 


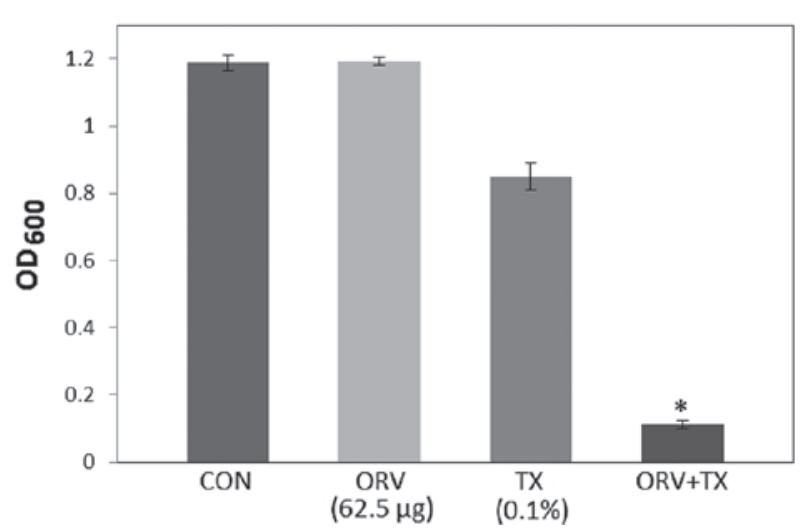

Figure 1. Effect of the membrane-permeabilizing agent, TX, on the susceptibility of Staphylococcus aureus (ATCC 33591) to ORV treatment. Bacteria viability was determined spectrophotometrically after reincubation for $36 \mathrm{~h}$ with $62.5 \mu \mathrm{g} / \mathrm{ml} \mathrm{ORV}$ and $0.1 \%$ TX. Data are represented as an average of three-independent experiments. " $\mathrm{P}<0.01$, vs. ORV alone. TX, Triton X-100; ORV, oxyresveratrol; $\mathrm{CON}$, control; $\mathrm{OD}_{600}$, optical density at $600 \mathrm{~nm}$.

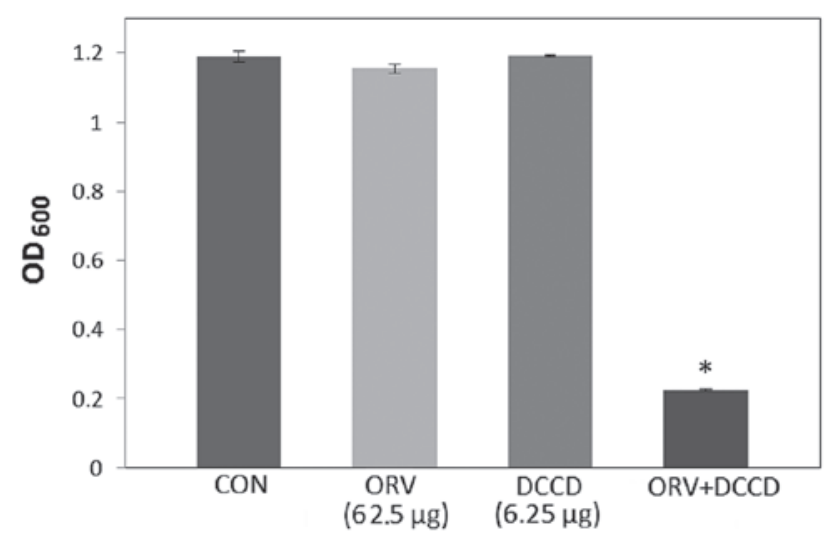

Figure 2. Effect of the ATPase-inhibitor DCCD on the susceptibility of Staphylococcus aureus (ATCC 33591) to ORV treatment. The viability of bacteria was determined spectrophotometrically $\left(\mathrm{OD}_{600}\right)$ after incubation for $36 \mathrm{~h}$ with $62.5 \mu \mathrm{g} / \mathrm{ml} \mathrm{ORV}$ and $6.25 \mu \mathrm{g} / \mathrm{ml}$ DCCD. Data are represented as an average of three independent experiments. ${ }^{*} \mathrm{P}<0.01$, vs. ORV alone. DCCD, $N, N^{\prime}$-dicyclohexylcarbodiimide; ORV, oxyresveratrol; CON, control; $\mathrm{OD}_{600}$, optical density at $600 \mathrm{~nm}$.

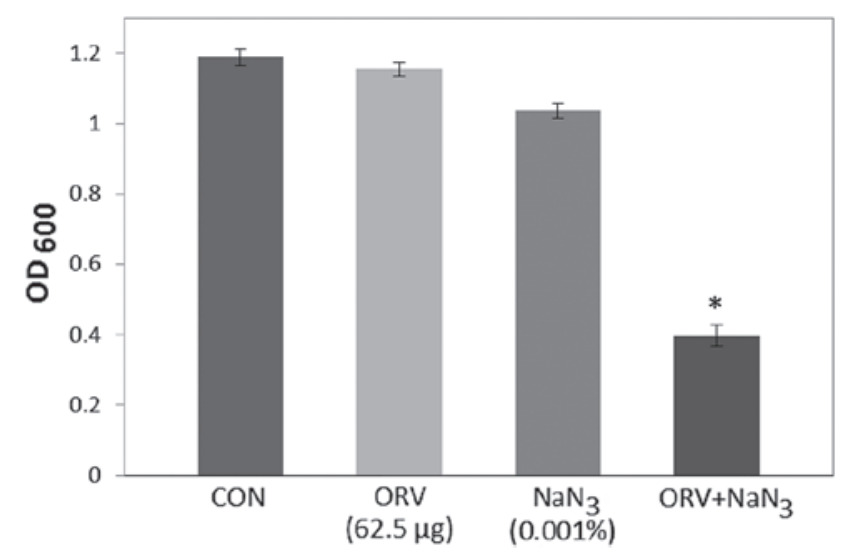

Figure 3. Effect of the ATPase-inhibitor $\mathrm{NaN}_{3}$ on the susceptibility of MRSA (ATCC 33591) to ORV. The viability of bacteria was determined spectrophotometrically $\left(\mathrm{OD}_{600}\right)$ after incubation for $24 \mathrm{~h}$ with $62.5 \mu \mathrm{g} / \mathrm{ml}$ ORV and $0.001 \% \mathrm{NaN}_{3}$. Data are the mean of three-independent experiments. "P $<0.01$, vs. ORV alone. $\mathrm{NaN}_{3}$, sodium azide; MRSA, MRSA, methicillin-resistant Staphylococcus aureus; ORV, oxyresveratrol; CON, control; $\mathrm{OD}_{600}$, optical density at $600 \mathrm{~nm}$.
The initial and most important step in reducing antibiotic resistance is to develop antibiotics from natural products that do not result in any toxic or other detrimental side effects $(1,26)$. The development of alternative antimicrobial drugs against infectious diseases is therefore required.

Our previous study demonstrated the synergistic effects of combining ORV with antibiotics in the treatment of MRSA, revealing that combinatorial treatment effectively inhibited MRSA growth (29). The present study aimed to develop anti-MRSA agents using novel combinations of ORV and antibiotics to directly address the resistance of MRSA bacteria. Antibacterial susceptibility tests, based on determination of cell proliferation, demonstrated the inhibitory effect of ORV against $S$. aureus compared with antibiotics AM, OX, VC, GT, NR, and CP. The results of the MIC assay performed on five strains of $S$. aureus are presented in Table II. The growth of MRSA was inhibited at $125 \mu \mathrm{g} / \mathrm{ml}$ ORV. The antibacterial activity of ORV had superior potency to the antibiotics AM and OX. The MICs of VC, GT, NR and CP were from $1.95-3.9 \mu \mathrm{g} / \mathrm{ml}$, from $62.5-250 \mu \mathrm{g} / \mathrm{ml}$, from $15.6-250 \mu \mathrm{g} / \mathrm{ml}$ and $31.25-500 \mu \mathrm{g} / \mathrm{ml}$, respectively.

To investigate the effects of enhanced membrane permeability and ATPase inhibition, the antibacterial activity of ORV was examined in the presence of a detergent (TX) and two ATPase-inhibiting agents (DCCD and $\mathrm{NaN}_{3}$ ). TX is a detergent known to increase the membrane permeability of various bacterial strains, and to induce the release of lipoteichoic acid (LTA) from the cell wall of $S$. aureus (30). LTA, a major constituent of the cell wall of gram-positive bacteria, is covalently bonded to the outer portion of PGN and is associated with the cytoplasmic membrane (23). TX has also been revealed to reduce methicillin resistance and increase antibiotic sensitivity in S. aureus strains (23).

In the present study, $S$. aureus was demonstrated to have an increased susceptibility to ORV in combination with TX, compared with that of ORV alone $(62.5 \mu \mathrm{g} / \mathrm{ml})$, as reported in Fig. 1. In addition, compared with the $\mathrm{OD}_{600}$ value for ORV alone $(62.5 \mu \mathrm{g} / \mathrm{ml})$, the $\mathrm{OD}_{600}$ value for the combination of TX with ORV was reduced by $89.8 \%$ (Fig. 1), while bacterial viability in the presence of ORV with DCCD (Fig. 2) and $0.001 \% \mathrm{NaN}_{3}$ (Fig. 3) was also reduced by $80.5 \%$ and $68.9 \%$, respectively. DCCD impedes the ATP-binding cassette transporter, whilst $\mathrm{NaN}_{3}$ is a metabolic inhibitor that reduces the ATP levels by disrupting the electrochemical proton gradients in the bacteria (21). In the present study, S. aureus viability was markedly reduced upon addition of ORV in combination with ATPase inhibitors (DCCD or $\mathrm{NaN}_{3}$ ), compared with the use of ORV alone (Figs. 2 and 3). These results demonstrate that the anti-MRSA activity of ORV is enhanced by changes in the membrane permeability and a reduced ATP level.

The cell wall of gram-negative bacteria typically contains $<10 \%$ of PGN, whereas the PGN content in gram-positive bacteria ranges from 5-95\% (31). The gram-positive bacterial cell wall consists of 30-50 PGN sheets outside the cell membrane, which is important in cell division and osmotic protection (32). ORV directly binds the cell wall and affects its integrity. In the present study, ORV-induced inhibition of bacterial growth (Fig. 4) indicated that ORV interfered with bacterial biosynthesis. PGN at a dose of $62.5 \mu \mathrm{g} / \mathrm{ml}$ completely blocked the antibacterial activity of ORV, indicating the direct 

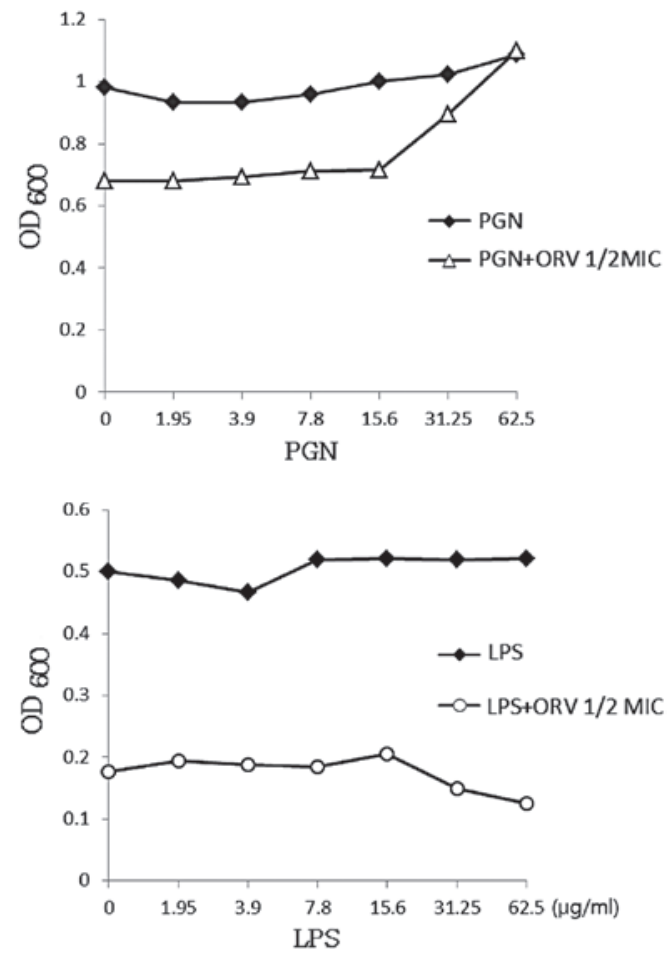

Figure 4. ORV-blocking effect by exogenous PGN of the S. aureus cell wall. LPS was used as a control. ORV, oxyresveratrol; PGN, peptidoglycan; LPS, lipopolysaccharide; MIC, minimum inhibitory concentration; $\mathrm{OD}_{600}$, optical density at $600 \mathrm{~nm}$.

binding of ORV with PGN on the cell wall. The cell wall ORV-binding effect of PGN was confirmed by the addition of PGN derived from S. aureus into MHB containing ORV alone $(62.5 \mu \mathrm{g} / \mathrm{ml})$. Under the same conditions, LPS, which was used as the control, did not demonstrate any such effect.

The cell morphology of ORV-treated cells was observed using TEM, which confirmed weakening of the cell wall and lytic effects of ORV on the S. aureus strain, ATCC 33591 (33). The micrographs reported in Fig. 5 illustrate the cell wall and membrane damage following ORV treatment in S. aureus. The control cells demonstrated normal S. aureus morphology with distinct septa (Fig. 5A), whereas bacterial cells treated with ORV at a dose of $62.5 \mu \mathrm{g} / \mathrm{ml}$ (i.e. $50 \%$ of the MIC) had a deformed septum and midline disruption (Fig. 5B). In addition, upon exposure of S. aureus to a higher dose of ORV (125 $\mu \mathrm{g} /$ $\mathrm{ml}$, i.e. the MIC), cell division and ghost cells were observed (Fig. 5C). Distinct septa were rarely discerned in the treated cells. TEM observation of ORV-treated MRSA cells substantiates the results indicating that ORV treatment induced altered expression of cell wall- and cell division-associated genes in MRSA.

In conclusion, the results of the present study suggests that ORV has antibacterial activity against MRSA. However, additional in vivo experiments are required to confirm that ORV may be effective against MRSA infections, which will be addressed in subsequent studies.

\section{Acknowledgements}

The present study was supported by grants from the Ministry of Food and Drug Safety (grant.no. 12172MFDS990; 2014),
A

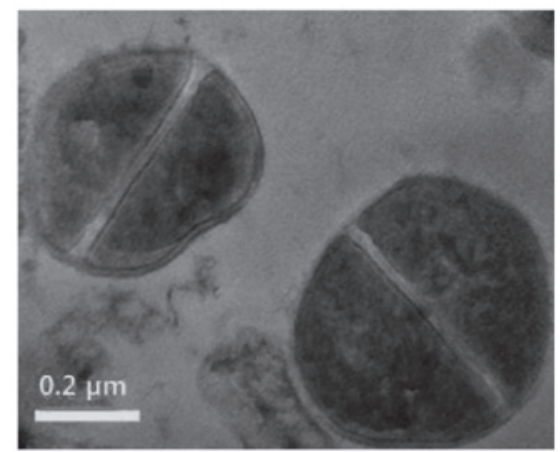

B

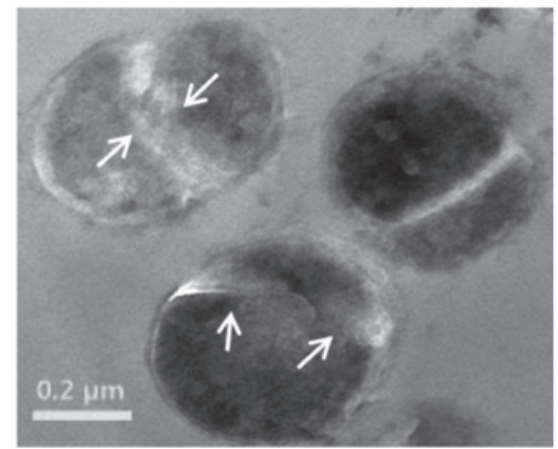

C

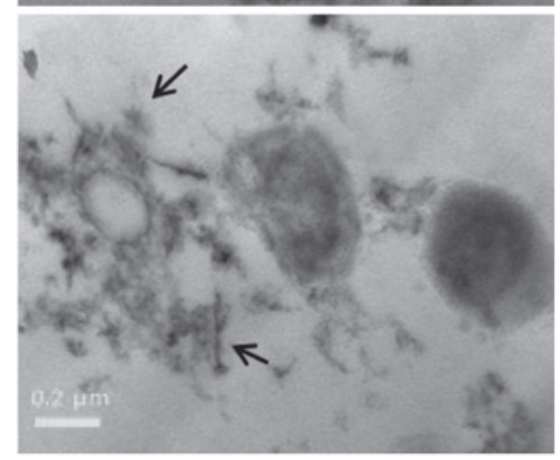

Figure 5. Transmission electron microscopy images of S. aureus (strain, ATCC 33591) after 24 h ORV treatment. (A) Untreated control S. aureus. Treatment of S. aureus with ORV at a dose of (B) $62.5 \mu \mathrm{g} / \mathrm{ml}(50 \%$ of the MIC), with white arrows indicating the cytoplasmic membrane disruption, and (C) $125 \mu \mathrm{g} / \mathrm{ml}$ (MIC), with black arrows indicating the separation of cytoplasmic contents from the membrane. ORV, oxyresveratrol; MIC, minimum inhibitory concentration.

and the National Research Foundation of Korea (grant. no. 2008-0062484; funded by the Korean government).

\section{References}

1. Mun SH, Kang OH, Joung DK, Kim SB, Choi JG, Shin DW and Kwon DY: In vitro anti-MRSA activity of carvone with gentamicin. Exp Ther Med 7: 891-896, 2014.

2. Whitby M, McLaws ML and Berry G: Risk of death from methicillin-resistant Staphylococcus aureus bacteraemia: A meta-analysis. Med J Aust 175: 264-267, 2001.

3. Bozic KJ and Ries MD: The impact of infection after total hiparthroplasty on hospital and surgeon resource utilization. J Bone Joint Surg Am 8: 1746-1751, 2005.

4. Gracia E, Fernández A, Conchello P, Laclériga A, Paniagua L, Seral F and Amorena B: Adherence of Staphylococcus aureus slime-producing strain variants to biomaterials used in orthopaedic surgery. Int Orthop 21: 46-51, 1997.

5. Seghrouchni K, van Delden C, Dominguez D, Benkabouche M, Bernard L, Assal M, Hoffmeyer P and Uçkay I: Remission after treatment of osteoarticular infections due to Pseudomonas aeruginosa versus Staphylococcus aureus: A case-controlled study. Int Orthop 36: 1065-1071, 2012. 
6. De Lucas-Villarrubia JC, Lopez-Franco M, Granizo JJ, De Lucas-Garcia JC and Gomez-Barrena E: Strategy to control methicillin resistant Staphylococcus aureus post-operative infection in orthopaedic surgery. Int Orthop 28: 16-20, 2004

7. Aqil F, Ahmad I and Owais M: Evaluation of anti-methicillin resistant Staphylococcus aureus (MRSA) activity and synergy of some bioactive plant extracts. Biotechnol J 1: 1093-1102, 2006.

8. Iqbal S, Younas U, Sirajuddin, Chan KW, Sarfraz RA and Uddin K: Proximate composition and antioxidant potential of leaves from three varieties of Mulberry (Morus sp.): A comparative study. Int J Mol Sci 13: 6651-6664, 2012.

9. Singab AN, El-Beshbishy HA, Yonekawa M, Nomura T and Fukai T: Hypoglycemic effect of Egyptian Morus alba root bark extract: Effect on diabetes and lipid peroxidation of streptozotocin-induced diabetic rats. J Ethnopharmacol 100: 333-338, 2005.

10. Jin WY, Na MK, An RB, Lee HY, Bae KH and Kang SS: Antioxidant compounds from twig of Morus alba. Nat Prod Sci 13: 129-132, 2002

11. Chung KO, Kim BY, Lee MH, Kim YR, Chung HY, Park JH and Moon JO: In-vitro and in-vivo anti-inflammatory effect of oxyresveratrol from Morus alba L. J Pharm Pharmacol 55: $1695-1700,2003$

12. Sritularak B, De-Eknamkul W and Likhitwitayawuid K: Tyrosinase inhibitors form Artocarpus lakoocha. Thai J Pharm Sci 22: 149-155, 1998.

13. Likhitwitayawuid K, Sritularak B and Benchanak K: Phenolics with antiviral activity from Millettia erythrocalyx and Artocarpus lakoocha. Nat Prod Res 19: 177-182, 2005.

14. Chuanasa T, Phromjai J, Lipipun V, Likhitwitayawuid K, Suzuki M, Pramyothin P, Hattori M and Shiraki K: Anti-herpes simplex virus (HSV-1) activity of oxyresveratrol derived from Thai medicinal plant: Mechanism of action and therapeutic efficacy on cutaneous HSV-1 infection in mice. Antiviral Res 80: 62-70, 2008

15. Lipipun V, Sasivimolphan P, Yoshida Y, Daikoku T, Sritularak B, Ritthidej G, Likhitwitayawuid K, Pramyothin P, Hattori M and Shiraki K: Topical cream-based oxyresveratrol in the treatment of cutaneous HSV-1 infection in mice. Antiviral Res 91: 154-160, 2011.

16. Sasivimolphan P, Lipipun V, Likhitwitayawuid K, Takemoto M, Pramyothin P, Hattori M and Shiraki K: Inhibitory activity of oxyresveratrol on wild-type and drug-resistant varicella-zoster virus replication in vitro. Antiviral Res 84: 95-97, 2009.

17. Kim YM, Yun J, Lee CK, Lee H, Min KR and Kim Y: Oxyresveratrol and hydroxystilbene compounds. Inhibitory effect on tyrosinase and mechanism of action. J Biol Chem 227: 16340-16344, 2002.

18. Lorenz P, Roychowdhury S, Engelmann M, Wolf G and Horn TF: Oxyresveratrol and resveratrol are potent antioxidants and free radical scavengers: Effect on nitrosative and oxidative stress derived from microglial cells. Nitric Oxide 9: 64-76, 2003.

19. Saowakon N, Tansatit T, Wanichanon C, Chanakul W, Reutrakul V and Sobhon P: Fasciola gigantica: Anthelmintic effect of the aqueous extract of Artocarpus lakoocha. Exp Parasitol 122: 289-298, 2009

20. Qiu F,Komatsu K, KawasakiK, Saito K, YaoX and Kano Y: Anovel stilbene glucoside, oxyresveratrol 3'-O-beta-glucopyranoside, from the root bark of Morus alba. Planta Med 62: 559-561, 1996.
21. Mun SH, Joung DK, Kim SB, Park SJ, Seo YS, Gong R, Choi JG, Shin DW, Rho JR, Kang OH and Kwon DY: The mechanism of antimicrobial activity of sophoraflavanone B against methicillin-resistant Staphylococcus aureus. Foodborne Pathog Dis 11: 234-239, 2014.

22. Clinical and Laboratory Standards Institute: Performance Standards for Antimicrobial Susceptibility Testing; Twenty-Fourth Informational Supplement. CLSI document M100-S24. Clinical and Laboratory Standards Institute, Wayne, PA, 2014

23. Cordwell SJ, Larsen MR, Cole RT and Walsh BJ: Comparative proteomics of Staphylococcus aureus and the response of methicillin-resistant and methicillin-sensitive strains to Triton X-100. Microbiology 148: 2765-2781, 2002.

24. Shibata H, Saito H, Yomota C, Kawanishi T and Okuda H: Alterations in the detergent-induced membrane permeability and solubilization of saturated phosphatidylcholine/cholesterol liposomes: Effects of poly(ethylene glycol)-conjugated lipid. Chem Pharm Bull (Tokyo) 60: 1105-1111, 2012.

25. Linnett PE and Beechey RB: Inhibitors of the ATP synthetase system. Methods Enzymol 55: 472-518, 1979.

26. Jung HJ and Lee DG: Synergistic antibacterial effect between silybin and N,N'-dicyclohexylcarbodiimide in clinical Pseudomonas aeruginosa isolates. J Microbiol 46: 462-467, 2008.

27. Zhao WH,HuZQ,OkuboS, Hara Y and Shimamura T: Mechanism of synergy between epigallocatechin gallate and beta-lactams against methicillin-resistant Staphylococcus aureus. Antimicrob Agents Chemother 45: 1737-1742, 2001.

28. Al-Habib A, Al-Saleh E, Safer AM and Afzal M: Bactericidal effect of grape seed extract on methicillin-resistant Staphylococcus aureus (MRSA). J Toxicol Sci 35: 357-364, 2010.

29. Joung DK, Choi SH, Kang OH, Kim SB, Mun SH, Seo YS, Kang DH, Gong R, Shin DW, Kim YC and Kwon DY: Synergistic effects of oxyresveratrol in conjunction with antibiotics against methicillin-resistant Staphylococcus aureus. Mol Med Rep 12: 663-667, 2015.

30. Komatsuzawa H, Ohta K, Sugai M, Fujiwara T, Glanzmann P, Berger-Bächi B and Suginaka H: Tn551-mediated insertional inactivation of the $\mathrm{fmtB}$ gene encoding a cell wall-associated protein abolishes methicillin resistance in Staphylococcus aureus. J Antimicrob Chemother 45: 421-431, 2000.

31. Farca AM, Nebbia P and Re G: Potentiation of antibiotic activity by EDTA-tromethamine against three clinically isolated gram-positive resistant bacteria. An in vitro investigation. Vet Res Commun 18: 1-6, 1994.

32. Lorian V and Atkinson B: Effect of serum on gram-positive cocci grown in the presence of penicillin. J Infect Dis 138: 865-871, 1978

33. Muthaiyan A, Martin EM, Natesan S, Crandall PG, Wilkinson BJ and Ricke SC: Antimicrobial effect and mode of action of terpeneless cold-pressed Valencia orange essential oil on methicillin-resistant Staphylococcus aureus. J Appl Microbiol 112: 1020-1033, 2012. 